\title{
Digital Predistortion of Millimeter-Wave Phased Array Transmitter With Over-the-air Calibrated Simplified Conductive Feedback Architecture
}

\author{
Nuutti Tervo, Bilal Khan, Olli Kursu, Janne P. Aikio, Markku Jokinen, Marko E. Leinonen, \\ Markku Juntti, Timo Rahkonen, and Aarno Pärssinen \\ University of Oulu, Faculty of Information Technology and Electrical Engineering (ITEE) \\ firstname.lastname@oulu.fi
}

\begin{abstract}
Digital predistortion (DPD) of a phased array requires that multiple transmit paths must be measured by a feedback (FB) receiver ( $\mathrm{Rx})$. This paper proposes a simplified FB architecture to be used for phased array DPD. A single FB line collects the waveform samples from the parallel paths to the FB Rx. The gain and phase of the common FB line is obtained by comparing the FB outputs to over-the-air (OTA) measurements. The overall DPD training is done by collecting the PA outputs with the common FB line, post-equalizing them to model the main lobe waveform and combining them to create the object used for array DPD. The DPD performance was verified by OTA measurements with 5 GNR waveform and $28 \mathrm{GHz} 8$-path phased array transmitter. The DPD trained through the local FB line with the proposed calibration method achieved $<-45 \mathrm{~dB}$ ACPR which was close to OTA DPD performance.

Keywords - Antenna array, Beamforming, DPD, Feedback Receiver, GaN, Linearization, OTA-calibration
\end{abstract}

\section{INTRODUCTION}

Fifth generation (5G) [1] and upcoming sixth generation (6G) [2] utilize high carrier frequencies for wireless communications. Highly directive antennas and analog beamforming are being used to implement directive communications with limited digital signal processing resources. High data-rate targets require either extremely high bandwidth or high spectral efficiency. In sub $6 \mathrm{GHz}$ frequencies, 256-quadrature amplitude modulation (QAM) and higher modulations are used, while in millimeter-wave $(\mathrm{mmW})$ frequencies (FR2 bands) 5G has specified modulations only up to 64-QAM. However, larger constellations are expected also in the FR2 bands in the near future.

High-order modulations and orthogonal frequency division multiplexing (OFDM) waveform set high linearity requirements for the phased array transmitters (Txs) in order to achieve low error vector magnitude (EVM). On the contrary, the adjacent channel power ratio (ACPR) specifications for $5 \mathrm{G}$ FR2 Tx are relatively loose [1], [3]. One way to improve the Tx linearity is to use digital predistortion (DPD). However, due to the analog beamforming architectures, multiple parallel nonlinear elements have to be linearized by a single DPD. Many different methods for phased array DPD are proposed in the literature and most of them aim for minimizing the radiated distortion. For achieving good EVM, the main target of the DPD is in the beamforming direction. Directive DPD has been proposed for example in [4]-[9].

DPD requires a feedback $(\mathrm{FB})$ receiver $(\mathrm{Rx})$ to measure the nonlinear output of the device under test (DUT). For array,

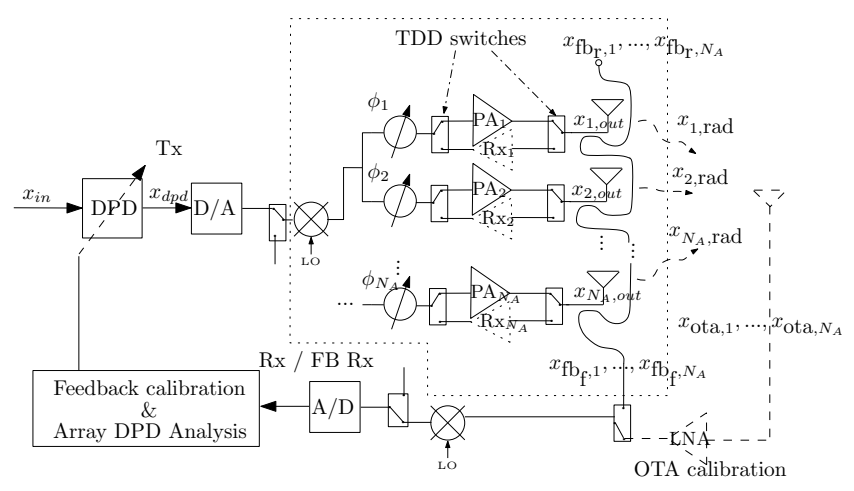

Fig. 1. Phased array Tx with proposed combined FB architecture and OTA calibration. The blocks inside the dotted box are implemented on the demonstration PCB.

the nonlinear output consists of multiple parallel elements. Hence, FB strategy plays a crucial role in the array DPD concept as the measurements are used to build the object for training the DPD. Several array FB architectures have been proposed in the literature. In general, they can be divided into conductive (e.g. [4]-[9], [10]-[11]), and over-the-air (OTA) (e.g. [3],[8],[12]-[14]) FB methods. OTA methods give low-complexity ways of measuring the radiated response. However, phase-coherent summation of the array outputs requires that the observation $\mathrm{Rx}$ antenna is placed in the far-field of the array that may be several meters away from the DUT. This may not be feasible in compact array designs used in $\mathrm{mmW}$ base stations and the far-field reference antenna may interfere with the beam transmitted by the array. Coupling antennas are used in [14], but they can effectively only collect the paths connected to the adjacent antenna elements.

The conductive FB architectures contain a single PA FB [10], switchable FB [4]-[5], [7] and combined anti-beamforming or phased FB [6], [11]. If the paths have different nonlinear characteristics, single PA FB is not enough to build a decent DPD object. On the contrary, the combined phased FB architectures have high complexity due to the additional analog beamforming performed in the FB path. Switchable FB architectures aim to collect each PA at different time instant. All state-of-the-art switchable FB architectures use additional switches to connect the FB $\mathrm{Rx}$ to the PA outputs one-by-one. In such a time sharing scheme it is also challenging to observe the phase of the output in a time-domain measurement. 
The FB architecture presented in this paper contains a FB line shared by multiple transmit paths. The concept is depicted in Fig. 1. The dotted line surrounds the content of the implemented printed circuit board (PCB). Time-division-duplex (TDD) switches of the individual Tx paths are used to enable and disable paths one-by-one. Compared to existing FB architectures, this offers an alternative way of training the DPD object without using additional switches or beamforming in the FB path. Amplitude and phase of the FB line from each coupling point to the FB output is post-equalized by comparing the FB measurements to the OTA calibration measurements. After the one-time FB calibration, the reference antenna is not required for DPD training any more.

\section{Phased Array Transmitter With OTA-CALIBRATED COMMON FEEDBACK LINE}

\section{A. $28 \mathrm{GHz}$ Transceiver Module with GaN PAs}

Photograph of the 16-chain $28 \mathrm{GHz}$ phased array transceiver (TRx) PCB and 64-element antenna array is shown in Fig. 2 and Fig. 3, respectively [15]-[17]. Each of the 16 paths is connected to a $2 \times 2$-element fixed unit cell via sub-miniature push-on micro (SMPM) bullets. The unit cells have $\lambda$ spacing at $28 \mathrm{GHz}$. In this paper, only half of the transmit paths are used and hence 8 Tx paths drive 32 patch antenna elements. The used elements are surrounded by the orange box in Fig. 3. The isolation between the individual antenna ports is better than $35 \mathrm{~dB}$ [17]. The antenna array is connected to the TRx via SMPM-bullets. The common transmit path of all paths consist of HMC264LC3B mixer and TGA2595 Gallium Nitride (GaN) PA. After the PA, the signal is divided into two antenna rows by a PD-0530SM Wilkinson power divider. Both rows have two TGA2595 PAs separated by controllable 5-bit HMC939 attenuators to drive the Wilkinson power division network. The individual paths contain TGP2100 5-bit phase shifters and TGA2595 PAs. The Tx and Rx paths are separated by MASW-011036 TRx switches such that $\mathrm{Tx}$ and $\mathrm{Rx}$ share the same phase shifters, power division network and mixer.

\section{B. Common Feedback Line}

The TRx contains two FB lines and one of them is used in this paper. Layout of one FB line is shown in Fig. 4. A meandering microstrip line is routed between SMPM antenna connectors and it couples to each PA output on the same side. Coupling is not directional as signals from individual PA output ports (ports 1 to 8 ) are coupled to both FB line ports (FB1 and FB2). For training individual transmit paths, FB1 is used to couple the signal transmitted to each antenna one by one. The EM simulated (ADS Momentum) coupling of the coupler is $\sim 40 \mathrm{~dB}$ from the PA output port to the FB line port. The simulated loss from the PA output port to the antenna connector is $\sim 1 \mathrm{~dB}$. A meandering microstrip line is used in the transmit paths to match the group delay from the common path to each antenna connector.

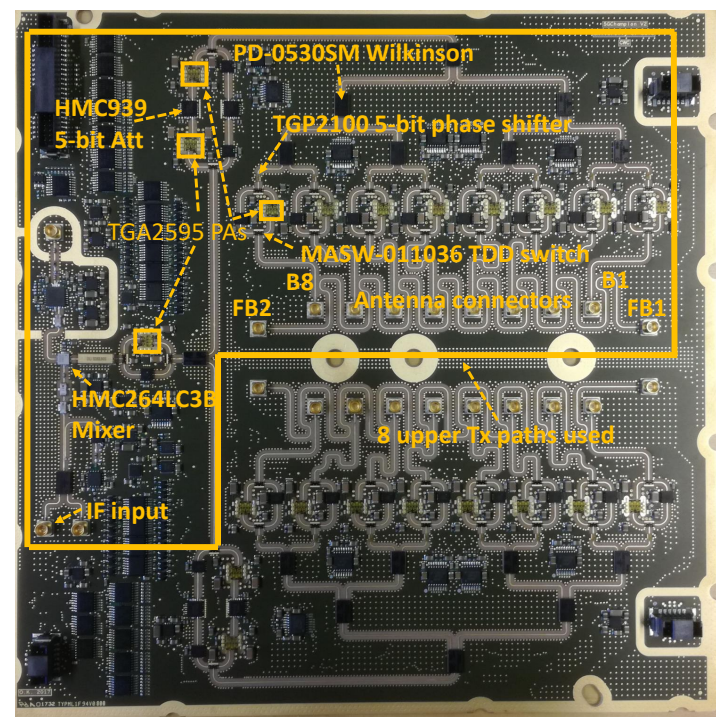

Fig. 2. Photograph of the $28 \mathrm{GHz}$ phased array Tx under test.

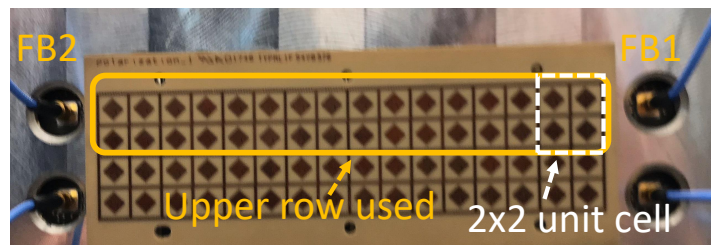

Fig. 3. 64-element antenna array.

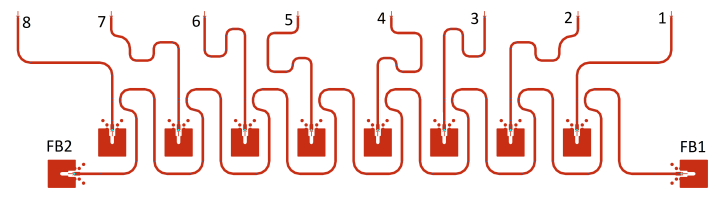

Fig. 4. Layout picture of the common FB line for eight transmit paths.

\section{Feedback Line Calibration by OTA-measurements}

The FB line architecture used for DPD has gain and phase (delay) differences from the individual coupling points to the FB output. Hence, the coupled response from each PA is slightly different. As the aim of the common DPD is to linearize the array in the far-field, the FB line was calibrated by OTA measurements. The calibration procedure transforms the FB output to the reference point in the far-field. The behavior of the FB is static and hence one-time FB calibration was performed. A flow chart of the calibration method is shown in Fig. 5. The TDD switches of the phased array are configured to enable each Tx path one-by-one while the other parallel PAs are terminated to $50 \mathrm{ohm}$. Each Tx path is measured from the FB output $x_{f b, 1}, \ldots, x_{f b, 8}$ and from the observation $\mathrm{Rx} x_{\text {ota,1 }}, \ldots, x_{\text {ota }, 8}$ placed at 1.5 meter distance from the array. The used distance satisfies the array far-field condition at the used center frequency of $28.1 \mathrm{GHz}$. A photograph of the measurement setup is presented in Fig. 6. A $100 \mathrm{MHz}$ wide 256-QAM cyclic prefix (CP)-OFDM signal is fed to the intermediate frequency (IF) input of the DUT 
by Keysight M8190A arbitrary waveform generator (ARB) and E8257B PSG. The OTA Rx and common FB output are connected to the Keysight N9040B UXA signal analyzer via Keysight custom-built switch box. In the OTA Rx, we use CA2630-141 pre-amplifier and A-info LB-28-15 standard gain horn antenna. The oversampling ratio used in the Rx was four. One should note that the OTA Rx and the switch box are only used to calibrate the feedback line and verify the radiated DPD performance. After the calibration, the OTA Rx can be disconnected from the system.

The phases of the FB signals are normalized against the common input signal $x_{i n}$ as

$$
x_{f b, i, n o r m}=x_{f b, i} \exp \left(-j \arg \left(\max \left(x_{f b, 1} \star x_{i n}\right)\right)\right),
$$

where $(\star)$ denotes the cross correlation between two signals, arg denotes the argument of a complex number and $j$ denotes the imaginary unit. The phases of the OTA measured reference signals are normalized also by the same method. The purpose of phase normalization is to model the coherent combining of the signals in the main lobe of the beam. The phase of the received waveform varies due to the measurement setup as the used time domain triggering cannot make two measurements measured with different time instants to be phase coherent. Hence, the setup cannot capture the actual carrier phase differences of the Tx paths used for analog beamforming. The finite impulse response (FIR) filters $w_{f, i}$ are fitted between the OTA measured reference signal and the FB signal by least squares (LS) method. The estimates of the modelled OTA FB signals are written as

$$
\hat{x}_{\text {ota }, i}=w_{f, i} * x_{f b, i, n o r m},
$$

where $\hat{x}_{o t a, i}$ denotes the estimate of the modelled OTA waveform from $i$ th TX path and $(*)$ denotes the convolution. Hence, each FIR aims of converting the FB signal to the signal modelled in the array far-field in the main lobe direction. The FB post-equalization includes also the impact of the individual antenna elements. Thus, the estimated main lobe signal from the common FB measurements can be written as

$$
\hat{x}_{\text {main lobe }}=\sum_{i=1}^{8} \hat{x}_{\text {ota }, i} \text {. }
$$

\section{Digital Predistortion of Phased Array with COMMON FEEDBACK LINE}

The modelled array far-field response calculated by (3) is used as the array output for the DPD training. As a DPD model, we use memory polynomial (MP) with memory depth $M=6$ and nonlinear order $K=9$ written as

$$
y_{M P}(n)=\sum_{m=1}^{M} \sum_{\substack{k=1 \\ k: \text { odd }}}^{K} d_{m k} x(n-m)|x(n-m)|^{k-1},
$$

where $d_{m k}$ denotes the $k$ th nonlinear order coefficient of $m$ th order lag. The DPD coefficients are solved by using LS method and the training is performed over 25000 samples. As a reference DPD, we use OTA trained DPD, where the

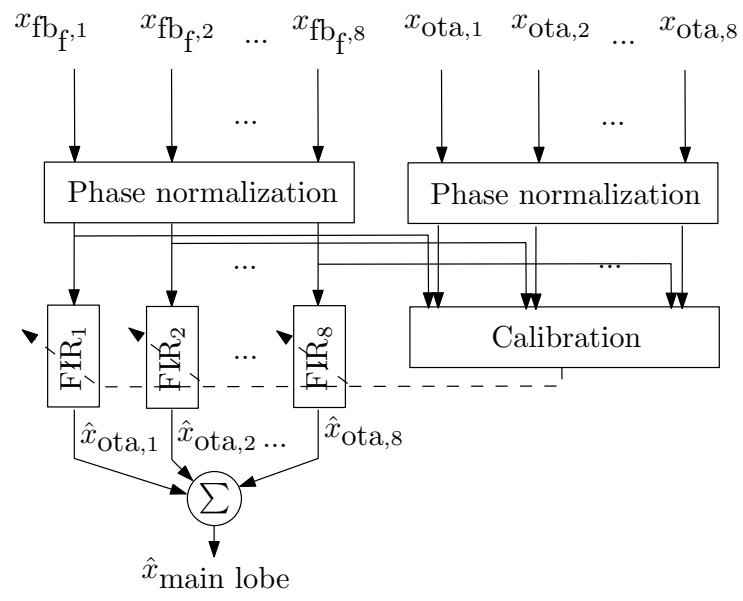

Fig. 5. Procedure for FB calibration and DPD object generation.

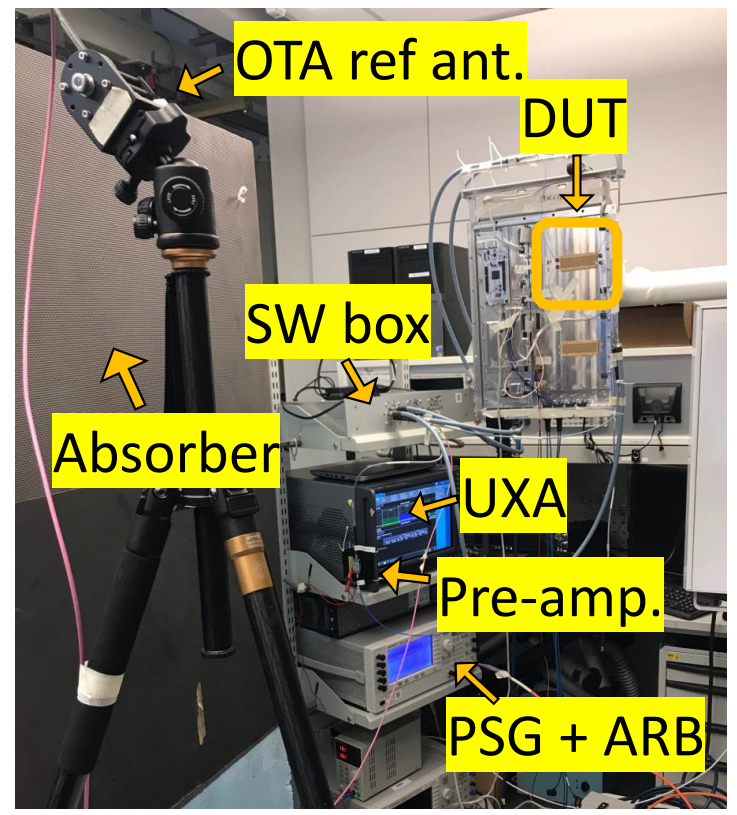

Fig. 6. Measurement setup for FB calibration and DPD demonstration.

complete array is trained with all elements active and the signal is measured from the main lobe direction. The same DPD model (4) is used for all cases.

The main lobe spectra measured from $0^{\circ}$ azimuth direction are depicted in Fig. 7. The figure consist of five plots which are (i), without DPD, (ii) DPD with only phase normalization in the FB, (iii) DPD with FB amplitude and phase equalization (1-tap FIR), (iv) DPD with 2-tap FIR FB post-equalization, and (v) DPD with OTA training. The corresponding ACPR results are collected to Table 1. FB equalization with higher number of taps did not give any better response and hence they were left out from the results. Already the DPD based on the phase normalized FB outputs gives good linearization performance. This is due to the fact that the nonlinearity of the individual paths is highly correlated as the PAs are driven with similar amplitude and there is no significant coupling over the antennas. However, the FB equalization with 1 and 2-tap FIRs 


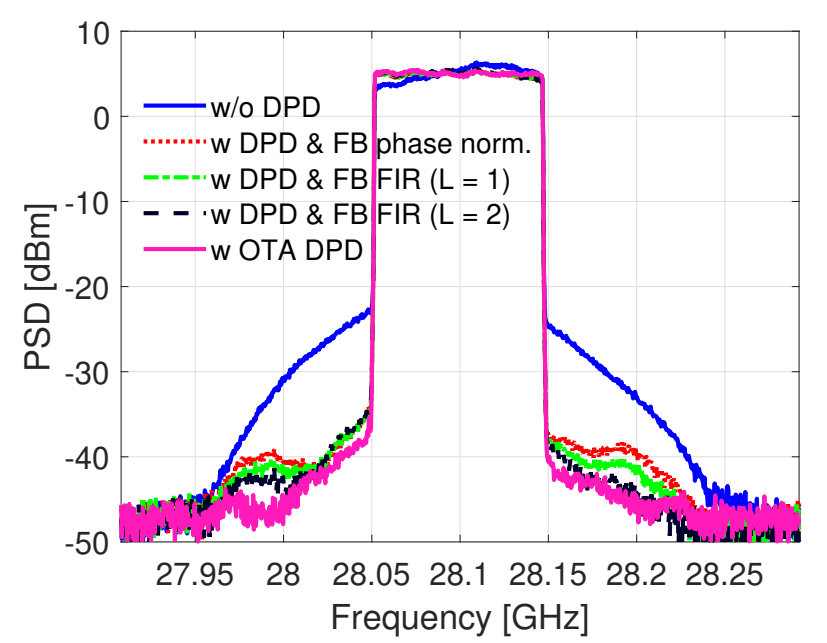

Fig. 7. OTA-measured main lobe spectra with and without DPD and FB post-equalization.

is able to improve the results even further. The OTA trained DPD used as a reference scenario gives the best performance but with only by a small margin compared to case (iv). The need for equalizing the FB paths from the coupling point to the common reference point is important especially when the Tx paths have significant differences in their nonlinear behavior. Such scenario may be for example when the PAs are driven with different amplitudes as in [4].

Table 1. OTA-measured ACPRs and EIRPs with and without DPD and FB post-equalization.

\begin{tabular}{|l|c|c|c|}
\hline FOM & EIRP [dBm] & ACPR $_{\mathrm{L}}[\mathrm{dB}]$ & $\mathrm{ACPR}_{\mathrm{H}}[\mathrm{dB}]$ \\
\hline w/o DPD & 31.3 & -33.5 & -35.4 \\
\hline $\begin{array}{l}\text { w DPD \& FB } \\
\text { phase norm. }\end{array}$ & 31.2 & -44.6 & -45.5 \\
\hline $\begin{array}{l}\text { w DPD \& FB eq } \\
\text { FIR (L=1) }\end{array}$ & 31.2 & -45.0 & -46.7 \\
\hline $\begin{array}{l}\text { w DPD \& FB eq } \\
\text { FIR (L=2) }\end{array}$ & 31.2 & -45.5 & -48.3 \\
\hline w OTA DPD & 31.2 & -47.5 & -49.2 \\
\hline
\end{tabular}

\section{CONCLUSION}

A common FB line architecture was proposed to be used for training the DPD by using the TDD switches of the array to enable individual array elements one-by-one. Compared to the existing FB architectures, the proposed one does not need a large switch matrix to connect the coupled signal from each PA output to the FB. The individual Tx paths can be trained in different TDD time slots during a dedicated training period. The common FB line was calibrated by OTA measurements to transfer each measured FB signal to model the OTA measured main lobe signal. The DPD performance was verified by OTA measurements and the DPD trained through the common FB line was able to give comparable results compared to the OTA-trained DPD. Better than $-45 \mathrm{dBc}$ main lobe ACPR was reached. The equalization of the feedback paths was concluded to be important especially when the individual Tx branches have different nonlinear characteristics.

\section{ACKNOWLEDGMENT}

This research has been supported by Business Finland 5G-VIIMA project, Academy of Finland 6Genesis Flagship (grant no. 318927) and Infotech Oulu Doctoral Programme.

\section{REFERENCES}

[1] 3GPP, "NR; Base Station (BS) radio transmission and reception (Release 15)," 3rd Generation Partnership Project (3GPP), https://www.3gpp.org/specifications, Technical Specification (TS) 38.104, 12 2018, version 15.2.0.

[2] M. Latva-aho and K. Leppänen, Key drivers and research challenges for $6 G$ ubiquitous wireless intelligence. Oulu, Finland: University of Oulu, 2019.

[3] N. Tervo et al., "Digital Predistortion Concepts for Linearization of mmW Phased Array Transmitters," in 2019 16th International Symposium on Wireless Communication Systems (ISWCS), Aug 2019, pp. 325-329.

[4] $\frac{}{2}$, "Digital Predistortion of Amplitude Varying Phased Array Utilising Over-the-air Combining," in 2017 IEEE MTT-S International Microwave Symposium (IMS), June 2017, pp. 1165-1168.

[5] X. Liu et al., "Beam-Oriented Digital Predistortion for 5G Massive MIMO Hybrid Beamforming Transmitters," IEEE Transactions on Microwave Theory and Techniques, vol. 66, no. 7, pp. 3419-3432, July 2018.

[6] M. Abdelaziz et al., "Digital Predistortion for Hybrid MIMO Transmitters," IEEE Journal of Selected Topics in Signal Processing, vol. 12, no. 3, pp. 445-454, June 2018.

[7] S. Hesami et al., "Single Digital Predistortion Technique for Phased Array Linearization," in 2019 IEEE International Symposium on Circuits and Systems (ISCAS), May 2019, pp. 1-5.

[8] E. Ng, Y. Beltagy, P. Mitran, and S. Boumaiza, "Single-Input Single-Output Digital Predistortion of Power Amplifier Arrays in Millimeter Wave RF Beamforming Transmitters," in 2018 IEEE/MTT-S International Microwave Symposium - IMS, June 2018, pp. 481-484.

[9] E. Ng et al., "Digital Predistortion of Millimeter-Wave RF Beamforming Arrays Using Low Number of Steering Angle-Dependent Coefficient Sets," IEEE Transactions on Microwave Theory and Techniques, vol. 67, no. 11, pp. 4479-4492, Nov 2019.

[10] L. Liu, W. Chen, L. Ma, and H. Sun, "Single-PA-feedback Digital Predistortion for Beamforming MIMO Transmitter," in 2016 IEEE International Conference on Microwave and Millimeter Wave Technology (ICMMT), vol. 2, June 2016, pp. 573-575.

[11] N. Tervo et al., "Analyzing the Effects of PA Variations on the Performance of Phased Array Digital Predistortion," in 2018 IEEE 29th Annual International Symposium on Personal, Indoor and Mobile Radio Communications (PIMRC), Sep. 2018, pp. 215-219.

[12] E. Ng, A. B. Ayed, P. Mitran, and S. Boumaiza, "Single-Input Single-Output Digital Predistortion of Multi-user RF Beamforming Arrays," in 2019 IEEE MTT-S International Microwave Symposium (IMS), June 2019, pp. 472-475.

[13] X. Liu et al., "Linearization for Hybrid Beamforming Array Utilizing Embedded Over-the-Air Diversity Feedbacks," IEEE Transactions on Microwave Theory and Techniques, pp. 1-14, 2019.

[14] X. Wang et al., "Real-Time Single Channel Over-the-Air Data Acquisition for Digital Predistortion of 5G Massive MIMO Wireless Transmitters," in 2019 IEEE MTT-S International Wireless Symposium (IWS), May 2019, pp. 1-3.

[15] M. E. Leinonen et al., " $28 \mathrm{GHz}$ Wireless Backhaul Transceiver Characterization and Radio Link Budget," ETRI Journal, vol. 40, no. 15, pp. 89-100, Feb. 2018.

[16] O. Kursu et al., "Design and Measurement of a 5G mmW Mobile Backhaul Transceiver at $28 \mathrm{GHz}$," EURASIP Journal on Wireless Communications and Networking, vol. 2018, no. 1, p. 201, Aug 2018.

[17] M. Sonkki et al., "Linearly Polarized 64-element Antenna Array for mm-wave Mobile Backhaul Application," in 12th European Conference on Antennas and Propagation (EuCAP 2018), April 2018, pp. 1-5. 